MATHEMATICS OF COMPUTATION

Volume 73, Number 248, Pages 1699-1718

S 0025-5718(03)01629-6

Article electronically published on December 19, 2003

\title{
GRAD-DIV STABLILIZATION FOR STOKES EQUATIONS
}

\author{
MAXIM A. OLSHANSKII AND ARNOLD REUSKEN
}

\begin{abstract}
In this paper a stabilizing augmented Lagrangian technique for the Stokes equations is studied. The method is consistent and hence does not change the continuous solution. We show that this stabilization improves the well-posedness of the continuous problem for small values of the viscosity coefficient. We analyze the influence of this stabilization on the accuracy of the finite element solution and on the convergence properties of the inexact Uzawa method.
\end{abstract}

\section{INTRODUCTION}

This paper provides an analysis of the effects of a particular stabilizing term that can be added to the Stokes equations. We consider the variational Stokes problem: given $f \in H^{-1}(\Omega)^{d}$, find $(u, p) \in H_{0}^{1}(\Omega)^{d} \times L_{2}(\Omega)$ with $\int_{\Omega} p(x) d x=0$ such that (1.1)

$$
\begin{aligned}
\nu(\nabla u, \nabla v)+\alpha(u, v)+\xi(\operatorname{div} u, \operatorname{div} v)+(\operatorname{div} v, p) & =f(v) & & \text { for } v \in H_{0}^{1}(\Omega)^{d}, \\
(\operatorname{div} u, q) & =0 & & \text { for } q \in L_{2}(\Omega),
\end{aligned}
$$

with parameters $\nu \in(0,1], \alpha \geq 0, \xi \geq 0$, where $(\cdot, \cdot)$ stands for the $L_{2}$ scalar product. To simplify the presentation, we restrict ourselves to the two cases $\alpha=0$, $\alpha=1$. In this paper we study the effects of the term (div $u, \operatorname{div} v)$ on the numerical solution of the Stokes problem. In the strong formulation this term is represented by the differential operator $\nabla$ div and, as we will show, adding this term has a stabilizing effect for small $\nu$ values. This explains why we call this a " $\nabla$ div stabilization". Note that the unique solution of problem (1.1) does not depend on $\xi$. Adding the consistent $\nabla$ div term to Stokes equations is not a new idea. This stabilization term is considered at several places in the literature. In 9 it is proposed and analyzed in the general framework of augmented Lagrangian methods. Indeed, in the saddle point formulation of the Stokes problem, adding the $\nabla$ div term results in an augmented Lagrangian. In [9] it is shown that this generally improves the rate of convergence of descent-type iterative methods for solving the saddle point problem. In 4], [10] the influence of the $\nabla$ div term on the convergence of iterative solvers for the Stokes and Navier-Stokes problems is studied. In [10] it is demonstrated that for the incompressible Navier-Stokes equations with high Reynolds numbers, the additional $\nabla$ div term improves the performance of nonlinear iterations. The analysis in [4] shows that for block-diagonal and block-triangular preconditioning of the Stokes problem this term does not lead to convergence improvement if it

Received by the editor November 7, 2001 and, in revised form, March 5, 2003.

2000 Mathematics Subject Classification. Primary 65N30, 65N22, 76D07.

Key words and phrases. Stokes equations, finite elements, augmented Lagrangian, inexact Uzawa. 
is used in the residual calculation only while not affecting the preconditioner. We note that in this paper we consider a preconditioner for the Stokes problem which depends on $\xi$.

In 21] it is shown that using this stabilization in the mixed formulation of the Poisson equation improves the rate of convergence of a suitably preconditioned MINRES method.

In the literature on the finite element method for incompressible Navier-Stokes equations, this term sometimes occurs in an error analysis of the streamline diffusion or Petrov-Galerkin finite element method (e.g., [15], 20]). From the analysis, however, it is not clear whether this term plays a key role or is introduced for technical reasons only.

In the papers mentioned $([9,4,21])$ the effect of the $\nabla$ div stabilization on the rate of convergence of iterative methods for solving the discretized Stokes equations has been studied. We observed that there are also interesting effects concerning the continuous problem and the quality of the finite element discretization and, to our knowledge, there is no theoretical explanation for this known in the literature. This paper tries to fill this gap. In practical implementations $\xi=0$ is the usual choice. In this paper we try to make clear when $\xi>0$ leads to a (significant) improvement.

We analyze the influence of the $\nabla$ div term on the continuous problem, the finite element discretization and the iterative solution of the discrete problem. Concerning the continuous problem, we show that although taking $\xi>0$ in (1.1) does not change the continuous solution, it has a clear positive effect on the stability of the bilinear form corresponding to (1.1). We will show that for $\xi>0$ the continuous problem is uniformly (w.r.t. $\nu$ ) well-posed in the natural energy norm. This is not true for the case $\xi=0$. The main new result presented in this paper concerns finite element error bounds for problem (1.1). We use LBB stable conforming finite element spaces for discretization and show that for $\nu \downarrow 0$ the discretization error bounds become significantly better if a $\nabla$ div stabilization is used. Numerical experiments show that the theoretical upper bounds predict the correct behaviour. We also analyze an Uzawa type iterative method for solving the discrete problem and draw a similar conclusion as in [9]; namely, the rate of convergence of the outer iteration for solving the Schur complement equation for the pressure in general increases due to the $\nabla$ div term. However, for $\nu \downarrow 0$, the inner velocity problem becomes more stiff if we use $\nabla$ div stabilization.

We will present results of numerical experiments which confirm our theoretical analysis. Based on the theory and the results of the experiments, our conclusion is that by adding the $\nabla$ div term the Stokes problem with $\nu \ll 1$ can be solved up to a prescribed accuracy with significantly lower arithmetic costs. We note that numerical experiments (in, e.g., [12]) have shown that similar positive effects of the $\nabla$ div stabilization occur in (linearized) Navier-Stokes equations with high Reynolds numbers.

As usual for a stabilization method, a proper value of the stabilization parameter, $\xi$ in our case, is important. In the present paper we only briefly address this issue. Results of numerical experiments related to this parameter choice can be found in [12.

Finally we have two remarks on scaling arguments. First, for the case $\alpha=0, \xi=$ 0 a common scaling argument using $\tilde{p}=\nu^{-1} p, \tilde{f}=\nu^{-1} f$ leads to a parameterindependent Stokes problem with a new pressure variable and right-hand side. One 
can then use known results for this Stokes problem (in $(u, \tilde{p})$ ) and transform back to the $(u, p)$ variables. For example, a typical discretization error bound of the form

$$
\left\|u-u_{h}\right\|_{1}+\left\|\tilde{p}-\tilde{p}_{h}\right\|_{0} \leq C h\left(\|u\|_{2}+\|\tilde{p}\|_{1}\right)
$$

immediately yields

$$
\left\|u-u_{h}\right\|_{1}+\frac{1}{\nu}\left\|p-p_{h}\right\|_{0} \leq C h\left(\|u\|_{2}+\frac{1}{\nu}\|p\|_{1}\right),
$$

with a constant $C$ that is independent of $\nu$. If, however, we take $\xi>0$, there are two parameters and the problem cannot be reduced to a parameter-independent standard Stokes problem by a simple scaling argument. One could use a scaling to eliminate one of the parameters. This then results in a parameter-dependent problem with scaled variables. We found that the analysis is most transparent if one does not apply a scaling but considers problem (1.1) in the original variables $(u, p)$. Therefore we will not use scaling arguments.

Secondly, another scaling argument relates problem (1.1) with $\alpha=1$ to a transient-like problem. Let $(u, p)$ be the solution of (1.1) with $\alpha=1$. Define

$$
\tilde{\xi}:=\frac{\xi}{\Delta t}, \quad \tilde{\nu}:=\frac{\nu}{\Delta t}, \quad \text { and } \quad \tilde{u}:=\Delta t u .
$$

Then the pair $(\tilde{u}, p)$ satisfies

$$
\begin{aligned}
\tilde{\nu}(\nabla \tilde{u}, \nabla v)+\frac{1}{\Delta t}(\tilde{u}, v)+\tilde{\xi}(\operatorname{div} \tilde{u}, \operatorname{div} v)+(\operatorname{div} v, p) & =f(v) & & \text { for } v \in H_{0}^{1}(\Omega)^{d} \\
(\operatorname{div} \tilde{u}, q) & =0 & & \text { for } q \in L_{2}(\Omega) .
\end{aligned}
$$

For the case $\xi=0$, this type of problem occurs if one applies an implicit time integration method (with a time step $\Delta t$ ) to a standard unsteady Stokes problem. Results for problem (1.1), like for example finite element discretization error bounds, immediately yield corresponding results for problem (1.3) (cf. Remark 44). These discretization error bounds are fairly sharp if one considers arbitrary $f \in H^{-1}(\Omega)^{d}$ and show a clear stabilizing effect due to the $\nabla$ div term. If, however, problem (1.3) corresponds to a time-discretized unsteady Stokes problem, the right-hand side $f$ has a special structure and our general bounds are too pessimistic. For such discrete unsteady problems, other techniques for analyzing the discretization error which take into account the evolutionary nature of the problem (as in [19, 7]) should be used. Our analysis does not yield satisfactory discretization error bounds for the unsteady case with a time step $\Delta t$ tending to zero. The results we obtain concerning the efficiency of the inexact Uzawa iterative solver for the finite element discretization of (1.3) are satisfactory, even for the case $\Delta t$ tending to zero (Remark 6).

The paper is organized as follows. In $\$ 2$ we present two simple linear algebra results that will be used further on. In $\$ 3$ we consider the variational formulation of the Stokes problem and show that adding the $\nabla$ div term leads to a uniformly (for $\nu \downarrow 0$ ) well-posed problem in a natural norm. Finite element discretization error bounds are presented in $\$ 4$ The convergence of the inexact Uzawa iterative method for solving the discrete problem is discussed in $\$ 5$ Finally in 96 some numerical results are presented that illustrate important effects of $\nabla$ div stabilization. 


\section{Preliminaries}

We first derive two elementary linear algebra results related to a matrix $\mathcal{A}$ of saddle point type:

$$
\begin{gathered}
\mathcal{A}=\left(\begin{array}{cc}
A & B^{T} \\
B & 0
\end{array}\right), \quad \mathbb{R}^{n \times n} \ni A=A^{T}>0, \\
B \in \mathbb{R}^{m \times n}, m<n, \operatorname{rank}(B)=m .
\end{gathered}
$$

We use the notation $\langle\cdot, \cdot\rangle$ for the euclidean scalar product. The euclidean norm is denoted by $\|\cdot\|$. We define the energy scalar product $\langle x, y\rangle_{A}=\langle A x, y\rangle$ and energy norm $\|x\|_{A}^{2}=\langle x, x\rangle_{A}$. The spectral condition number of a regular matrix $C$ is denoted by $\kappa(C)=\|C\|\left\|C^{-1}\right\|$. The following quantities will play an important role

$$
\sqrt{\Gamma}:=\sup _{y \in \mathbb{R}^{m}, x \in \mathbb{R}^{n}} \frac{\langle B x, y\rangle}{\|x\|_{A}\|y\|}, \quad \sqrt{\gamma}:=\inf _{y \in \mathbb{R}^{m}} \sup _{x \in \mathbb{R}^{n}} \frac{\langle B x, y\rangle}{\|x\|_{A}\|y\|} .
$$

Here and in the remainder we always take $\inf _{x}$ or $\sup _{x}$ over nonzero elements. The following elementary result is known in the literature (see [22]). For completeness we also show a proof.

Lemma 2.1. Let $\lambda_{\min }\left(B A^{-1} B^{T}\right)$ and $\lambda_{\max }\left(B A^{-1} B^{T}\right)$ be the smallest and largest eigenvalues of the Schur complement $B A^{-1} B^{T}$. Then the following holds:

$$
\gamma=\lambda_{\min }\left(B A^{-1} B^{T}\right), \quad \Gamma=\lambda_{\max }\left(B A^{-1} B^{T}\right) .
$$

Proof. Note that

$$
\begin{aligned}
\sup _{x} \frac{\langle B x, y\rangle^{2}}{\|x\|_{A}^{2}\|y\|^{2}} & =\sup _{x} \frac{\left\langle B A^{-\frac{1}{2}} x, y\right\rangle^{2}}{\|x\|^{2}\|y\|^{2}}=\sup _{x} \frac{\left\langle x, A^{-\frac{1}{2}} B^{T} y\right\rangle^{2}}{\|x\|^{2}\|y\|^{2}} \\
& =\frac{\left\|A^{-\frac{1}{2}} B^{T} y\right\|^{2}}{\|y\|^{2}}=\frac{\left\langle B A^{-1} B^{T} y, y\right\rangle}{\langle y, y\rangle} .
\end{aligned}
$$

Hence

$$
\begin{aligned}
& \gamma=\inf _{y} \frac{\left\langle B A^{-1} B^{T} y, y\right\rangle}{\langle y, y\rangle}=\lambda_{\min }\left(B A^{-1} B^{T}\right), \\
& \Gamma=\sup _{y} \frac{\left\langle B A^{-1} B^{T} y, y\right\rangle}{\langle y, y\rangle}=\lambda_{\max }\left(B A^{-1} B^{T}\right) .
\end{aligned}
$$

Using this lemma we derive an elementary result concerning the spectral condition number of a preconditioned version of the matrix $\mathcal{A}$. Similar results are known in the literature (e.g., the equality (2.5) can be found in [1]).

Lemma 2.2. Define

$$
\mathcal{P}:=\left(\begin{array}{cc}
A^{-\frac{1}{2}} & 0 \\
0 & I
\end{array}\right) \mathcal{A}\left(\begin{array}{cc}
A^{-\frac{1}{2}} & 0 \\
0 & I
\end{array}\right)=\left(\begin{array}{cc}
I & A^{-\frac{1}{2}} B^{T} \\
B A^{-\frac{1}{2}} & 0
\end{array}\right) .
$$

Assume that $\gamma>0$ holds. Then $\mathcal{P}$ is invertible and

$$
\begin{aligned}
\|\mathcal{P}\| & =\frac{1}{2}(\sqrt{1+4 \Gamma}+1), \\
\left\|\mathcal{P}^{-1}\right\| & =\frac{2}{\min \{2, \sqrt{1+4 \gamma}-1\}} .
\end{aligned}
$$


Proof. With $C:=B A^{-\frac{1}{2}}$ we obtain

$$
\mathcal{P}=\left(\begin{array}{cc}
I & C^{T} \\
C & 0
\end{array}\right)
$$

Note that $C$ has a nontrivial kernel. For $v \in \operatorname{Ker}(C), v \neq 0$, we have $\mathcal{P}\left(\begin{array}{l}v \\ 0\end{array}\right)=\left(\begin{array}{l}v \\ 0\end{array}\right)$, hence $1 \in \sigma(\mathcal{P})$. For $\mu \in \sigma(\mathcal{P}), \mu \neq 1$, we have

$$
\left(\begin{array}{cc}
I & C^{T} \\
C & 0
\end{array}\right)\left(\begin{array}{l}
v_{1} \\
v_{2}
\end{array}\right)=\mu\left(\begin{array}{l}
v_{1} \\
v_{2}
\end{array}\right), \quad \text { with } v_{2} \neq 0
$$

This holds iff $\mu(\mu-1) \in \sigma\left(C C^{T}\right)$. Let $\lambda_{1} \leq \lambda_{2} \leq \ldots \leq \lambda_{m}$ be the eigenvalues of $C C^{T}=B A^{-1} B^{T}$. We then obtain

$$
\sigma(\mathcal{P}) \backslash\{1\}=\left\{\frac{1}{2}\left(1 \pm \sqrt{1+4 \lambda_{j}}\right) \mid 1 \leq j \leq m\right\} .
$$

Hence, the largest eigenvalue of $\mathcal{P}$ is given by

$$
\|\mathcal{P}\|=\frac{1}{2}\left(1+\sqrt{1+4 \lambda_{m}}\right)=\frac{1}{2}(1+\sqrt{1+4 \Gamma}) .
$$

Due to Lemma 2.1 and the assumption $\gamma>0$ we have that $\lambda_{1}>0$ holds. Hence $\mathcal{P}$ is invertible and the largest eigenvalue of the inverse is given by

$$
\begin{aligned}
\left\|\mathcal{P}^{-1}\right\| & =\max \left\{1, \max _{1 \leq j \leq m} 2\left|1 \pm \sqrt{1+4 \lambda_{j}}\right|^{-1}\right\} \\
& =\max \left\{1,2\left|1-\sqrt{1+4 \lambda_{1}}\right|^{-1}\right\} \\
& =\frac{2}{\min \{2, \sqrt{1+4 \gamma}-1\}}
\end{aligned}
$$

These results show that the quantities $\gamma$ and $\Gamma$ completely determine $\kappa\left(B A^{-1} B^{T}\right)$ and $\kappa(\mathcal{P})$. Note that the former depends only on the quotient $\Gamma / \gamma$, whereas for the latter this is not the case. The result in Lemma 2.2 will be used below.

\section{The continuous Stokes equations}

We consider a standard variational formulation of the Stokes problem in a domain $\Omega$ in $d$-dimensional Euclidean space $(d=2,3)$. We use the notations

$$
X:=H_{0}^{1}(\Omega)^{d}, \quad M:=\left\{f \in L^{2}(\Omega) \mid \int_{\Omega} f(x) d x=0\right\} .
$$

The $L^{2}$ scalar product and associated norm are denoted by $(\cdot, \cdot),\|\cdot\|$, respectively. Before we turn to the Stokes problem in (1.1), we first consider a more general setting. We introduce two continuous bilinear forms:

$$
a(\cdot, \cdot): X \times X \rightarrow \mathbb{R}, \quad b(\cdot, \cdot): X \times M \rightarrow \mathbb{R} .
$$

We assume that $a(\cdot, \cdot)$ is symmetric and $X$-elliptic and that the bilinear form $b$ satisfies the infsup condition

$$
\inf _{q \in M} \sup _{v \in X} \frac{b(v, q)}{\|\nabla v\|\|q\|} \geq \beta>0 .
$$


We consider the standard saddle point problem: given $f \in X^{\prime}$, find $(u, p) \in X \times M$ such that

$$
\left\{\begin{aligned}
a(u, v)+b(v, p) & =f(v) \quad \text { for } v \in X \\
b(u, q) & =0 \quad \text { for } q \in M
\end{aligned}\right.
$$

Using the bilinear form $\phi:(X \times M) \times(X \times M) \rightarrow \mathbb{R}$,

$$
\phi(u, p ; v, q):=a(u, v)+b(v, p)+b(u, q),
$$

problem (3.3) can be rewritten as follows. Find $(u, p) \in X \times M$ such that

$$
\phi(u, p ; v, q)=f(v) \text { for all }(v, q) \in X \times M .
$$

On $X$ we introduce the norm induced by the bilinear form $a$ : $\|u\|_{X}:=a(u, u)^{\frac{1}{2}}$ for $u \in X$. On $M$ we use the $L^{2}$-norm $\|\cdot\|$ and on the product space we use the norm

$$
|\|(u, p)\||=\left(\|u\|_{X}^{2}+\|p\|^{2}\right)^{\frac{1}{2}} .
$$

We introduce the notation

$$
\sqrt{\Gamma}:=\sup _{v \in X, q \in M} \frac{b(v, q)}{\|v\|_{X}\|q\|}, \quad \sqrt{\gamma}:=\inf _{q \in M} \sup _{v \in X} \frac{b(v, q)}{\|v\|_{X}\|q\|} .
$$

Note that $\Gamma, \gamma$ are used in (2.2) to denote similar quantities. Below the symbols $\Gamma, \gamma$ always refer to the quantities in (3.5). The infsup condition (3.2) implies $\gamma>0$. We emphasize that if the bilinear forms $a(\cdot, \cdot)$ and $b(\cdot, \cdot)$ correspond to the Stokes problem (as in (3.9) below), then the infsup constant $\beta$ from (3.2) does not depend on any parameters, whereas $\Gamma$ and $\gamma$ depend on the parameters $\nu, \alpha$ and $\xi$. The quantities $\Gamma$ and $\gamma$ completely determine the continuity and stability of the bilinear form $\phi$, as shown below.

Theorem 3.1. For all $(u, p),(v, q) \in X \times M$ we have

$$
|\phi(u, p ; v, q)| \leq \frac{1}{2}(\sqrt{1+4 \Gamma}+1)|\|(u, p)||||||(v, q) \mid\|
$$

and

$$
\sup _{(v, q) \in X \times M} \frac{\phi(u, p ; v, q)}{|\|(v, q)\||} \geq \frac{1}{8} \min \{1, \gamma\}|\|(u, p)\|| .
$$

Proof. We define $\theta:=\frac{1}{2}(\sqrt{1+4 \Gamma}+1)$ and note that

$$
\begin{aligned}
|\phi(u, p ; v, q)| & =|a(u, v)+b(v, p)+b(u, q)| \\
& \leq\|u\|_{X}\|v\|_{X}+\Gamma^{\frac{1}{2}}\|v\|_{X}\|p\|+\Gamma^{\frac{1}{2}}\|u\|_{X}\|q\| \\
& \leq\left(\|u\|_{X}^{2}+\frac{\Gamma}{\theta}\|u\|_{X}^{2}+\theta\|p\|^{2}\right)^{\frac{1}{2}}\left(\|v\|_{X}^{2}+\frac{\Gamma}{\theta}\|v\|_{X}^{2}+\theta\|q\|^{2}\right)^{\frac{1}{2}} \\
& =\theta\|\|(u, p)\|\|\|(v, q)\| .
\end{aligned}
$$

This proves the result in (3.6).

For $(f, g) \in X^{\prime} \times M^{\prime}$, let $(u, p) \in X \times M$ be the solution of

$$
\phi(u, p ; v, q)=f(v)+g(q) \quad \text { for all }(v, q) \in X \times M \text {. }
$$

The mapping $(f, g) \rightarrow(u, p)$ is bijective. A standard analysis (e.g., in [8] §4.1, [14] § 7.4.1) yields the following sharp bounds on the norms of $u$ and $p$ :

$$
\begin{aligned}
\|u\|_{X} & \leq\|f\|_{X^{\prime}}+2 \gamma^{-\frac{1}{2}}\|g\|_{M^{\prime}}, \\
\|p\| & \leq \gamma^{-\frac{1}{2}}\left(\|f\|_{X^{\prime}}+\|u\|_{X}\right) \leq 2 \gamma^{-\frac{1}{2}}\left(\|f\|_{X^{\prime}}+\gamma^{-\frac{1}{2}}\|g\|_{M^{\prime}}\right) .
\end{aligned}
$$


Hence

$$
\begin{aligned}
|\|u, p\|| & \leq\|u\|_{X}+\|p\| \leq 2\left(1+\gamma^{-\frac{1}{2}}\right)\left(\|f\|_{X^{\prime}}+\gamma^{-\frac{1}{2}}\|g\|_{M^{\prime}}\right) \\
& =2\left(1+\gamma^{-\frac{1}{2}}\right)\left(\sup _{v \in X} \frac{f(v)}{\|v\|_{X}}+\gamma^{-\frac{1}{2}} \sup _{q \in M} \frac{g(q)}{\|q\|}\right) \\
& \leq 4\left(1+\gamma^{-\frac{1}{2}}\right) \max \left\{1, \gamma^{-\frac{1}{2}}\right\} \sup _{(v, q) \in X \times M} \frac{f(v)+g(q)}{\|v\|_{X}+\|q\|} \\
& \leq 4\left(1+\gamma^{-\frac{1}{2}}\right) \max \left\{1, \gamma^{-\frac{1}{2}}\right\} \sup _{(v, q) \in X \times M} \frac{\phi(u, p ; v, q)}{\|\|(v, q) \|} .
\end{aligned}
$$

Let $z=\gamma^{-\frac{1}{2}} \in(0, \infty)$. A simple computation yields

$$
\frac{1}{4(1+z) \max \{1, z\}} \geq \frac{1}{8 \max \left\{1, z^{2}\right\}}=\frac{1}{8} \min \left\{1, z^{-2}\right\} .
$$

This proves the result in (3.7).

The result in (2.4) shows that the bound in (3.6) is sharp. The inverse of the infsup constant in (3.7) behaves like $\mathcal{O}\left(\gamma^{-1}\right)$ for $\gamma \rightarrow 0$. The same behaviour $\left\|\mathcal{P}^{-1}\right\|=\mathcal{O}\left(\gamma^{-1}\right)$ for $\gamma \rightarrow 0$ is observed in (2.5). In this sense, the result in (3.7) is sharp, too.

The results in Theorem 3.1 show that the condition number

$$
C(\gamma, \Gamma):=\frac{4(\sqrt{1+4 \Gamma}+1)}{\min \{1, \gamma\}}
$$

can be used as a measure for the well-posedness of the continuous problem (3.4) in the norm $|\|\cdot\||$.

We now consider the Stokes problem (1.1) with $\alpha \in\{0,1\}$. Note that the unique solution of this problem does not depend on $\xi$, since $\operatorname{div} v \in M$ for all $v \in X$. Continuity and stability results for problem (1.1) are known in the literature (e.g., 8). However, in the literature the parameters $\nu$ and $\xi$ are then treated as fixed constants (usually $\xi=0$ ). Here we allow these parameters to vary and analyze the the dependence of the condition number (i.e., the well-posedness) on the parameters $\nu$ and $\xi$. Hence, for the bilinear forms $a$ and $b$ in (3.3), we now take

$$
\begin{aligned}
a(u, v) & :=\nu(\nabla u, \nabla v)+\alpha(u, v)+\xi(\operatorname{div} u, \operatorname{div} v) \text { for } u, v \in X, \\
b(u, q) & :=(\operatorname{div} u, q) \quad \text { for } u \in X, q \in M .
\end{aligned}
$$

Note that $b$ satisfies the infsup condition (3.2) and $a$ is symmetric and $X$-elliptic. The norm $\|\cdot\|_{X}$ depends on the parameters $\nu, \alpha, \xi$. In the next theorem we describe the dependence of the condition number $C(\gamma, \Gamma)$ on the parameters $\nu, \alpha, \xi$. We use $\|\operatorname{div} u\| \leq\|\nabla u\|$ for $u \in X$ and the Friedrichs inequality

$$
\|u\| \leq c_{F}\|\nabla u\| \quad \text { for all } u \in X .
$$

Theorem 3.2. The following holds:

$$
\begin{aligned}
& C(\gamma, \Gamma) \leq \frac{4(\sqrt{5}+1)}{\beta^{2}} \frac{\max \left\{\beta^{2}, \nu+\xi\right\}}{\min \{1, \sqrt{\nu+\xi}\}}=: C_{0}(\nu, \xi) \quad \text { if } \alpha=0, \\
& C(\gamma, \Gamma) \leq \frac{4(\sqrt{5}+1)}{\beta^{2}} \frac{\max \left\{\beta^{2}, \nu+c_{F}^{2}+\xi\right\}}{\min \{1, \sqrt{\nu+\xi}\}}=: C_{1}(\nu, \xi) \quad \text { if } \alpha=1,
\end{aligned}
$$

with $\beta$ defined in (3.2). 
Proof. We take $\alpha \in[0,1]$. From

$$
\|u\|_{X}^{2} \leq\left(\nu+\alpha c_{F}^{2}+\xi\right)\|\nabla u\|^{2}
$$

and the infsup property (3.2) we obtain

$$
\gamma \geq \frac{\beta^{2}}{\nu+\alpha c_{F}^{2}+\xi}
$$

Using

$$
\|u\|_{X}^{2} \geq(\nu+\xi)\|\operatorname{div} u\|^{2}
$$

and the Cauchy-Schwarz inequality yields

$$
\Gamma \leq \frac{1}{\nu+\xi} .
$$

Using the inequality $\sqrt{1+4 x}+1 \leq(\sqrt{5}+1) \max \{1, \sqrt{x}\}$ we obtain

$$
\begin{aligned}
4 \frac{\sqrt{1+4 \Gamma}+1}{\min \{1, \gamma\}} & \leq 4(\sqrt{5}+1) \frac{\max \left\{1, \Gamma^{\frac{1}{2}}\right\}}{\min \{1, \gamma\}} \leq 4(\sqrt{5}+1) \frac{\max \left\{1, \frac{1}{\sqrt{\nu+\xi}}\right\}}{\min \left\{1, \frac{\beta^{2}}{\nu+\alpha c_{F}^{2}+\xi}\right\}} \\
& =\frac{4(\sqrt{5}+1)}{\beta^{2}} \frac{\max \left\{\beta^{2}, \nu+\alpha c_{F}^{2}+\xi\right\}}{\min \{1, \sqrt{\nu+\xi}\}} .
\end{aligned}
$$

Taking $\alpha \in\{0,1\}$ yields the bounds in (3.10) and (3.11) .

Corollary 1. We consider a few interesting cases.

- $\alpha=0, \xi=0$. The function $\nu \rightarrow C_{0}(\nu, 0)$ behaves like $\nu^{-\frac{1}{2}}$ for $\nu \downarrow 0$ and hence is unbounded for $\nu \downarrow 0$.

- $\alpha=0, \xi=\xi_{0}>0$. The function $\nu \rightarrow C_{0}\left(\nu, \xi_{0}\right)$ is bounded for $\nu \downarrow 0$, hence the problem is uniformly well-posed in the norm

$$
|\|(u, p)\||=\left(\nu\|\nabla u\|^{2}+\xi_{0}\|\operatorname{div} u\|^{2}+\|p\|^{2}\right)^{\frac{1}{2}} .
$$

- $\alpha=1, \xi=0$. The function $\nu \rightarrow C_{1}(\nu, 0)$ is unbounded for $\nu \downarrow 0$.

- $\alpha=1, \xi=\xi_{0}>0$. The bound is controlled for $\nu \downarrow 0$, hence we have uniform well-posedness in the norm

$$
|\|(u, p)\||=\left(\nu\|\nabla u\|^{2}+\|u\|^{2}+\xi_{0}\|\operatorname{div} u\|^{2}+\|p\|^{2}\right)^{\frac{1}{2}} .
$$

From these results we see that adding the term ( $\operatorname{div} u, \operatorname{div} v)$ in the variational Stokes problem makes the problem well-posed in the corresponding natural norm ||$|(\cdot, \cdot)|||$ uniformly for $\nu \in(0,1]$. Although adding the $(\operatorname{div} u, \operatorname{div} v)$ term does not change the solution of the Stokes problem, it yields robust (i.e., uniform w.r.t. $\nu$ ) stability bounds.

Remark 1. For $\alpha=1$ and $\nu \downarrow 0$, the Stokes problem is singularly perturbed. As a well-posed limit $(\nu=0, \xi=0)$ problem one can take the mixed formulation of the Poisson equation with Neumann boundary conditions. This limit problem in variational form uses the space $H(\operatorname{div}) \times M(M$ as in (3.1) $)$, with norm

$$
(u, p) \rightarrow\left(\|u\|^{2}+\|\operatorname{div} u\|^{2}+\|p\|^{2}\right)^{\frac{1}{2}} .
$$

In the analysis of the Stokes problem we use the norm

$$
|\|(u, p)\||=\left(\nu\|\nabla u\|^{2}+\|u\|^{2}+\xi\|\operatorname{div} u\|^{2}+\|p\|^{2}\right)^{\frac{1}{2}} .
$$


Note that in the limit case $\nu=0$ the latter norm is equivalent to the norm in (3.16) only for $\xi>0$. In this sense the $\nabla$ div term is a natural stabilizing term for the Stokes problem if $\nu \downarrow 0$.

Remark 2. For the case $\alpha=0, \nu \downarrow 0, \xi=0$, uniform well-posedness with respect to a special norm can be proved. It is well known that the standard Stokes problem, (1.1) with $\nu=1, \xi=\alpha=0$, is well-posed in the norm $(u, \tilde{p}) \rightarrow\left(\|\nabla u\|^{2}+\|\tilde{p}\|^{2}\right)^{\frac{1}{2}}$ on $X \times M$. A scaling argument as discussed in the introduction then immediately yields uniform well-posedness in the (anisotropic) norm

$$
\|\|(u, p)\|\|_{*}:=\left(\|\nabla u\|^{2}+\frac{1}{\nu^{2}}\|p\|^{2}\right)^{\frac{1}{2}} .
$$

It can be shown, using a similar analysis as presented above, that for the corresponding conditon number $C_{*}(\gamma, \Gamma)$ the uniform bound

$$
\left.\left.C_{*}(\gamma, \Gamma) \leq 4 \frac{\sqrt{1+4 \frac{\Gamma}{\nu}}+1}{\min \{1, \nu \gamma\}} \leq C \quad \text { for } \nu \in\right] 0,1\right],
$$

holds. The norm $|\|\cdot\||_{*}$ in (3.18) has a stronger anisotropy than the norm in (3.14), and using the latter results in better control of the velocity variable as $\nu \downarrow 0$.

\section{Finite Element Discretization Using GRAD-DiV STABilization}

We now consider the discretization of the variational Stokes problem using a family of pairs of LBB stable finite element spaces $X_{h} \subset X, M_{h} \subset M$ indexed by some mesh size parameter $h$. In this section we use standard arguments to derive a sharp discretization error bound and we show that for $\nu \downarrow 0$ taking $\xi=\xi_{0}>0$ instead of $\xi=0$ has a clear stabilizing effect.

Before we turn to the discrete Stokes problem we first consider the Galerkin discretization of the more general variational problem (3.3) or, equivalently, (3.4). We assume that the finite element pair $\left(X_{h}, M_{h}\right)$ is LBB stable with a constant $\hat{\beta}$ independent of $h$ :

$$
\inf _{q_{h} \in M_{h}} \sup _{v_{h} \in X_{h}} \frac{b\left(v_{h}, q_{h}\right)}{\left\|\nabla v_{h}\right\|\left\|q_{h}\right\|} \geq \hat{\beta}>0 .
$$

The discrete problem is as follows: find $\left(u_{h}, p_{h}\right) \in X_{h} \times M_{h}$ such that

$$
\phi\left(u_{h}, p_{h} ; v_{h}, q_{h}\right)=f\left(v_{h}\right) \quad \text { for all }\left(v_{h}, q_{h}\right) \in X_{h} \times M_{h} .
$$

We introduce the discrete analog of the quantity $\gamma$ :

$$
\sqrt{\gamma_{h}}:=\inf _{q_{h} \in M_{h}} \sup _{v_{h} \in X_{h}} \frac{b\left(v_{h}, q_{h}\right)}{\left\|v_{h}\right\| X\left\|q_{h}\right\|} .
$$

From (4.1) it follows that $\gamma_{h}>0$ holds. For the analysis below we introduce the formulation of the discrete problem as a linear system in $\mathbb{R}^{n+m}$. For this we assume standard bases in $X_{h}$ and $M_{h}$ and the corresponding isomorphisms

$$
J_{X}: \mathbb{R}^{n} \rightarrow X_{h}, n:=\operatorname{dim}\left(X_{h}\right), \quad J_{M}: \mathbb{R}^{m} \rightarrow M_{h}, m:=\operatorname{dim}\left(M_{h}\right) .
$$


Let the stiffness matrices $A \in \mathbb{R}^{n \times n}, B \in \mathbb{R}^{m \times n}$, and the mass matrix $\hat{M} \in \mathbb{R}^{m \times m}$ be given by

$$
\begin{aligned}
\langle A x, y\rangle & =a\left(J_{X} x, J_{X} y\right) \text { for all } x, y \in \mathbb{R}^{n}, \\
\langle B x, y\rangle & =b\left(J_{X} x, J_{M} y\right) \text { for all } x \in \mathbb{R}^{n}, y \in \mathbb{R}^{m}, \\
\langle\hat{M} x, y\rangle & =\left(J_{M} x, J_{M} y\right) \text { for all } x, y \in \mathbb{R}^{m} .
\end{aligned}
$$

We now prove an infsup property of $\phi$ on $X_{h} \times M_{h}$.

Lemma 4.1. The following holds:

$$
\begin{gathered}
\inf _{\left(u_{h}, p_{h}\right) \in X_{h} \times M_{h}} \sup _{\left(v_{h}, q_{h}\right) \in X_{h} \times M_{h}} \frac{\phi\left(u_{h}, p_{h} ; v_{h}, q_{h}\right)}{\left|\|\left(u_{h}, p_{h}\right)\right|||||\left|\left(v_{h}, q_{h}\right)\right|||} \\
=\frac{1}{2} \min \left\{2, \sqrt{1+4 \gamma_{h}}-1\right\} .
\end{gathered}
$$

Proof. With $\tilde{B}:=\hat{M}^{-\frac{1}{2}} B$, we obtain

$$
\begin{aligned}
\sqrt{\gamma_{h}} & :=\inf _{y \in \mathbb{R}^{m}} \sup _{x \in \mathbb{R}^{n}} \frac{\langle B x, y\rangle}{\langle A x, x\rangle^{\frac{1}{2}}\langle\hat{M} y, y\rangle^{\frac{1}{2}}} \\
& =\inf _{y \in \mathbb{R}^{m}} \sup _{x \in \mathbb{R}^{n}} \frac{\langle\tilde{B} x, y\rangle}{\|x\|_{A}\|y\|} .
\end{aligned}
$$

Let $L:=\left(\begin{array}{cc}I & A^{-\frac{1}{2}} \tilde{B}^{T} \\ \tilde{B} A^{-\frac{1}{2}} & 0\end{array}\right)$. Note that

$$
\begin{aligned}
& \inf _{\left(u_{h}, p_{h}\right) \in X_{h} \times M_{h}} \sup _{\left(v_{h}, q_{h}\right) \in X_{h} \times M_{h}} \frac{\phi\left(u_{h}, p_{h} ; v_{h}, q_{h}\right)}{\left\|( u _ { h } , p _ { h } ) \left|\left\|\left|\left\|\left(v_{h}, q_{h}\right) \mid\right\|\right.\right.\right.\right.} \\
= & \inf _{z \in \mathbb{R}^{n+m}} \sup _{w \in \mathbb{R}^{n+m}} \frac{\left\langle\left(\begin{array}{cc}
I & A^{-\frac{1}{2}} B^{T} \hat{M}^{-\frac{1}{2}} \\
\hat{M}^{-\frac{1}{2}} B A^{-\frac{1}{2}} & 0
\end{array}\right) z, w\right\rangle}{\|z\|\|w\|} \\
= & \inf _{z \in \mathbb{R}^{n+m}} \sup _{w \in \mathbb{R}^{n+m}} \frac{\langle L z, w\rangle}{\|z\|\|w\|}=\inf _{z \in \mathbb{R}^{n+m}} \frac{\|L z\|}{\|z\|}=\left\|L^{-1}\right\|^{-1} .
\end{aligned}
$$

We now apply Lemma 2.2.

Due to the continuity result (3.6) and the infsup result in the previous lemma we can prove a discretization error bound using standard arguments.

Theorem 4.2. Let $(u, p)$ be the solution of the continuous problem (3.4) and $\left(u_{h}, p_{h}\right)$ be the solution of the discrete problem (4.2). The following holds:

$$
\begin{aligned}
\left|\left\|\left(u-u_{h}, p-p_{h}\right) \mid\right\|\right. & \leq\left(1+\hat{C}\left(\gamma_{h}, \Gamma\right)\right) \min _{v_{h} \in X_{h}, q_{h} \in M_{h}}\left|\left\|\left(u-v_{h}, p-q_{h}\right) \mid\right\|,\right. \\
\text { with } \hat{C}\left(\gamma_{h}, \Gamma\right) & :=\frac{\sqrt{1+4 \Gamma}+1}{\min \left\{2, \sqrt{1+4 \gamma_{h}}-1\right\}} .
\end{aligned}
$$

Proof. For arbitrary $v_{h} \in X_{h}, q_{h} \in M_{h}$, define $e:=u-v_{h}, e_{h}=u_{h}-v_{h}, g:=$ $p-q_{h}, g_{h}:=p_{h}-q_{h}$. The Galerkin orthogonality property yields

$$
\phi\left(e_{h}, g_{h} ; w_{h}, r_{h}\right)=\phi\left(e, g ; w_{h}, r_{h}\right) \quad \text { for all }\left(w_{h}, r_{h}\right) \in X_{h} \times M_{h} \text {. }
$$


Using this in combination with the continuity and infsup results, we obtain, for suitable $\left(w_{h}, r_{h}\right) \in X_{h} \times M_{h}$,

$$
\begin{aligned}
\left\|\left(e_{h}, g_{h}\right) \mid\right\| & \leq \frac{2}{\min \left\{2, \sqrt{1+4 \gamma_{h}}-1\right\}} \frac{\phi\left(e_{h}, g_{h} ; w_{h}, r_{h}\right)}{\left|\left\|\left(w_{h}, r_{h}\right) \mid\right\|\right.} \\
& =\frac{2}{\min \left\{2, \sqrt{1+4 \gamma_{h}}-1\right\}} \frac{\phi\left(e, g ; w_{h}, r_{h}\right)}{\left|\left\|\left(w_{h}, r_{h}\right) \mid\right\|\right.} \\
& \leq \frac{\sqrt{1+4 \Gamma}+1}{\min \left\{2, \sqrt{1+4 \gamma_{h}}-1\right\}}|\|(e, g) \mid\| .
\end{aligned}
$$

Now combine this with the triangle inequality $\left|\left\|\left(u-u_{h}, p-p_{h}\right)\left|\left\|\leq\left|\left\|\left(e_{h}, g_{h}\right) \mid\right\|+\right.\right.\right.\right.\right.$ ||$|(e, g)| \|$.

We now consider the Stokes problem; i.e., in the remainder of this section the bilinear forms $a$ and $b$ are as in (3.9). Note that the bilinear form $a(\cdot, \cdot)$ depends on $\xi$ and that opposite to the continuous problem the discrete solution in general depends on $\xi$, unless $\operatorname{div} v_{h} \in M_{h}$ for all $v_{h} \in X_{h}$. Thus if one uses a space $X_{h}$ of divergence-free finite elements, the discrete solution does not depend on $\xi$. For the Stokes problem we have, for all $(u, p) \in X \times M$

$\frac{1}{2}\left(\nu^{\frac{1}{2}}\|\nabla u\|+\xi^{\frac{1}{2}}\|\operatorname{div} u\|+\alpha^{\frac{1}{2}}\|u\|+\|p\|\right) \leq|\|(u, p)\|| \leq\left(\nu^{\frac{1}{2}}+\xi^{\frac{1}{2}}\right)\|\nabla u\|+\alpha^{\frac{1}{2}}\|u\|+\|p\|$.

Using this in combination with Theorem 4.2 yields the discretization error bound

$$
\begin{aligned}
\nu^{\frac{1}{2}}\left\|\nabla\left(u-u_{h}\right)\right\|+\xi^{\frac{1}{2}}\left\|\operatorname{div}\left(u-u_{h}\right)\right\|+\alpha^{\frac{1}{2}}\left\|u-u_{h}\right\| & +\left\|p-p_{h}\right\| \\
\leq 2\left(1+\hat{C}\left(\gamma_{h}, \Gamma\right)\right)\left(\operatorname { m i n } _ { v _ { h } \in X _ { h } } \left\{\left(\nu^{\frac{1}{2}}+\xi^{\frac{1}{2}}\right)\left\|\nabla\left(u-v_{h}\right)\right\|\right.\right. & \left.+\alpha^{\frac{1}{2}}\left\|u-v_{h}\right\|\right\} \\
& \left.+\min _{q_{h} \in M_{h}}\left\|p-q_{h}\right\|\right) .
\end{aligned}
$$

We now analyze the dependence of the factor $\hat{C}\left(\gamma_{h}, \Gamma\right)$ on the parameters $\nu, \alpha, \xi$ and the mesh size parameter $h$.

Theorem 4.3. The following holds:

$$
\begin{aligned}
& \hat{C}\left(\gamma_{h}, \Gamma\right) \leq \frac{1}{4 \hat{\beta}^{2}}(\sqrt{5}+1)^{2} \frac{\max \left\{\hat{\beta}^{2}, \nu+\xi\right\}}{\min \{1, \sqrt{\nu+\xi}\}}=: \hat{C}_{0}(\nu, \xi) \quad \text { if } \alpha=0, \\
& \hat{C}\left(\gamma_{h}, \Gamma\right) \leq \frac{1}{4 \hat{\beta}^{2}}(\sqrt{5}+1)^{2} \frac{\max \left\{\hat{\beta}^{2}, \nu+c_{F}^{2}+\xi\right\}}{\min \{1, \sqrt{\nu+\xi}\}}=: \hat{C}_{1}(\nu, \xi) \quad \text { if } \alpha=1 .
\end{aligned}
$$

Proof. We take $\alpha \in[0,1]$. Note that for $x \geq 0$,

$$
\begin{aligned}
& \sqrt{1+4 x}+1 \leq(\sqrt{5}+1) \max \{1, \sqrt{x}\}, \\
& \sqrt{1+4 x}-1 \geq(\sqrt{5}-1) \min \{1, x\} .
\end{aligned}
$$

Hence,

$$
\hat{C}\left(\gamma_{h}, \Gamma\right) \leq \frac{1}{4}(\sqrt{5}+1)^{2} \frac{\max \{1, \sqrt{\Gamma}\}}{\min \left\{1, \gamma_{h}\right\}}
$$

holds. Using the Friedrichs inequality, we obtain

$$
\left\|u_{h}\right\|_{X}=\nu\left\|\nabla u_{h}\right\|^{2}+\alpha\left\|u_{h}\right\|^{2}+\xi\left\|\operatorname{div} u_{h}\right\|^{2} \leq\left(\nu+\alpha c_{F}^{2}+\xi\right)\left\|\nabla u_{h}\right\|^{2} .
$$


From the LBB property it follows that

$$
\gamma_{h} \geq \frac{\hat{\beta}^{2}}{\nu+\alpha c_{F}^{2}+\xi} .
$$

From (3.13) we have the bound

$$
\Gamma \leq \frac{1}{\nu+\xi} .
$$

Using the results (4.10) and (4.11) in (4.9) and taking $\alpha \in\{0,1\}$ yields the results in (4.7), (4.8).

The bounds for $\hat{C}\left(\gamma_{h}, \Gamma\right)$ in Theorem 4.3 are of the same form as the bounds for $C(\gamma, \Gamma)$ in Theorem 3.2 . Hence the results in Corollary 1 apply here; i.e., $\hat{C}_{0}(\nu, 0)$ and $\hat{C}_{1}(\nu, 0)$ are unbounded for $\nu \downarrow 0$, whereas for $\xi_{0}>0$ the factors $\hat{C}_{0}\left(\nu, \xi_{0}\right)$ and $\hat{C}_{1}\left(\nu, \xi_{0}\right)$ are uniformly bounded for $\nu \downarrow 0$. Due to Theorem 4.2, this has direct consequences for the discretization error bounds. To make this more clear we consider a concrete finite element pair. As an example we take the LBB stable pair of conforming $\mathrm{P} 1$ isoP2/P0 finite elements (piecewise linear velocity on a refined grid / piecewise constant for the pressure). We use standard approximation properties of these spaces and assume that the solution $(u, p)$ of the Stokes problem is sufficiently regular. We use the notation $\|\cdot\|_{k}$ for the norm on the Sobolev space $H^{k}(\Omega)(k=1,2)$. The results in (4.6) and Theorem 4.3 yield the following discretization error bounds for $\nu \in(0,1]$ :

- For $\alpha=0, \xi=0$,

$$
\nu^{\frac{1}{2}}\left\|\nabla\left(u-u_{h}\right)\right\|+\left\|p-p_{h}\right\| \leq C \nu^{-\frac{1}{2}} h\left(\nu^{\frac{1}{2}}\|u\|_{2}+\|p\|_{1}\right) .
$$

- For $\alpha=0, \xi=1$,

$$
\nu^{\frac{1}{2}}\left\|\nabla\left(u-u_{h}\right)\right\|+\left\|\operatorname{div}\left(u-u_{h}\right)\right\|+\left\|p-p_{h}\right\| \leq C h\left(\|u\|_{2}+\|p\|_{1}\right) .
$$

- For $\alpha=1, \xi=0$,

$$
\nu^{\frac{1}{2}}\left\|\nabla\left(u-u_{h}\right)\right\|+\left\|u-u_{h}\right\|+\left\|p-p_{h}\right\| \leq C \nu^{-\frac{1}{2}} h\left(\nu^{\frac{1}{2}}\|u\|_{2}+\|u\|_{1}+\|p\|_{1}\right) .
$$

- For $\alpha=1, \xi=1$,

(4.15) $\nu^{\frac{1}{2}}\left\|\nabla\left(u-u_{h}\right)\right\|+\left\|\operatorname{div}\left(u-u_{h}\right)\right\|+\left\|u-u_{h}\right\|+\left\|p-p_{h}\right\| \leq C h\left(\|u\|_{2}+\|p\|_{1}\right)$.

Note that for small $\nu$ the bounds for the case with $\nabla$ div-stabilization $(\xi=1)$ are significantly better than for the case $\xi=0$. These bounds indicate that the larger the $H^{1}$-norm of the pressure is compared to the $H^{2}$-norm of the velocity, the more important the stabilizing $\nabla$ div term is. Also note that for the case with $\nabla$ divstabilization, the term $\left\|\operatorname{div}\left(u-u_{h}\right)\right\|$ is controlled, wheras for $\xi=0$ this is not the case.

Remark 3. For the case $\alpha=0, \xi=0$, the scaling argument discussed in the introduction immediately yields a (sharp) discretization error bound. For the $\mathrm{P} 1$ isoP2/P0 finite elements this results in (cf. (1.2))

$$
\left\|\nabla\left(u-u_{h}\right)\right\|+\nu^{-1}\left\|p-p_{h}\right\| \leq C h\left(\|u\|_{2}+\nu^{-1}\|p\|_{1}\right) .
$$

For small $\nu$ values this bound is better than the one in (4.12) but worse than the result for the problem with $\nabla$ div-stabilization in (4.13). 
Remark 4. Using the scaling $\tilde{\xi}=\frac{\xi}{\Delta t}, \tilde{\nu}=\frac{\nu}{\Delta t}, \tilde{u}=\Delta t u$, the results for $\alpha=1$ in (4.14) and (4.15) immediately yield corresponding results for the transient-like Stokes problem in (1.3). Let $\left(\tilde{u}_{h}, p_{h}\right)$ be the discrete solution that results from the Galerkin discretization using P1isoP2/P0 finite element spaces applied to (1.3). The results in 4.14), (4.15) can be reformulated as

$$
\begin{aligned}
& \tilde{\nu}^{\frac{1}{2} \|} \nabla\left(\tilde{u}-\tilde{u}_{h}\right)\left\|+\frac{1}{\sqrt{\Delta t}}\right\| \tilde{u}-\tilde{u}_{h}\|+\sqrt{\Delta t}\| p-p_{h} \| \\
& \quad \leq \begin{cases}C \frac{h}{\sqrt{\tilde{\nu} \Delta t}}\left(\tilde{\nu}^{\frac{1}{2}}\|\tilde{u}\|_{2}+\frac{1}{\sqrt{\Delta t}}\|\tilde{u}\|_{1}+\sqrt{\Delta t}\|p\|_{1}\right), & \text { if } \xi=0, \\
C h\left(\frac{1}{\sqrt{\Delta t}}\|\tilde{u}\|_{2}+\sqrt{\Delta t}\|p\|_{1}\right) & \text { if } \xi=1 .\end{cases}
\end{aligned}
$$

From the numerical experiments in $\sqrt{6}$ it can be seen that the results in (4.14) and (4.15) are fairly sharp and hence the results in (4.17) are sharp, too. These results, however, are too pessimistic for $\Delta t \ll 1$ in the context of unsteady problems. As was pointed out in the introduction, to obtain more reliable error bounds, other techniques, which take into account the evolutionary nature of the problem, should be used.

Remark 5. Clearly if we introduce the $\nabla$ div term, we have to chose a reasonable value for the parameter $\xi$. Numerical experiments have shown that the effect of the stabilization is not very sensitive with respect to this choice, although $\xi$ should not be too large. An indication for a reasonable value can be obtained as follows. Assume that $\min _{v_{h} \in X_{h}}\left\|\nabla\left(u-v_{h}\right)\right\| \sim \min _{q_{h} \in M_{h}}\left\|p-q_{h}\right\|$ (for our example of $\mathrm{P} 1$ isoP2/P0 FE this is the case if $\|u\|_{2} \sim\|p\|_{1}$ ), and assume that $\alpha=0$ and $\nu$ is sufficiently small (actually $\nu \leq \hat{\beta}^{2}$ already suffices). Then the balance between velocity and pressure terms in the right-hand side of (4.6) is preserved if $\xi=O(1)$. The constant $\hat{C}$ in (4.6) also depends on $\xi$. The choice $\xi=\hat{\beta}^{2}$ minimizes $\hat{C}$. Moreover, under certain assumptions on the domain $\Omega$ and triangulation it is known that $\hat{\beta}^{2}=O(1)$ holds (cf. [6]). Therefore, with the above assumptions, the choice $\xi \sim \hat{\beta}^{2}$ is reasonable. Numerical experiments presented in [12] with common benchmark problems (driven cavity and backward facing step) show that for $\xi \in[0.1,0.2]$ one obtains good results. Note that for a unit square and P1isoP2/P0 FE, we have $\hat{\beta} \approx 0.44$.

\section{Preconditioning the Discrete problem}

In this section we discuss the iterative solution of the discrete problem (4.2). We restrict ourselves to iterative methods of inexact Uzawa type. For this class of methods applied to the stationary Stokes problem, convergence analyses are known (e.g., [1, 3]). Also for other iterative methods based on conjugate or minimal residual techniques there are convergence analyses available (cf. [16, [18] and the references therein). In all these analyses one assumes fixed values for $\nu$ and $\xi$, usually $\xi=0$, and one does not analyze the dependence of the convergence behaviour on variation in these parameters. Below, for the inexact Uzawa method we study how the rate of convergence depends on variation in the parameters $\nu, \xi$, and $\alpha$.

The effect of adding the term $(\operatorname{div} u, \operatorname{div} v)$ on the convergence speed of gradient type methods for solving the saddle point problem associated to (4.2) is analyzed in [9]. In the terminology of [9] the addition of the term $(\operatorname{div} u, \operatorname{div} v)$ yields a corresponding augmented Lagrangian. In [9] it is shown that gradient type of methods, like the exact Uzawa method, have a higher rate of convergence when applied to 
the problem with an augmented Lagrangian. In this section we will draw a similar conclusion for the inexact Uzawa method.

Finally note that for the case $\nu=1, \xi=0, \alpha>0$ (the transient-like Stokes problem), robustness results concerning the convergence of Uzawa-type methods are given in [2], 11].

We consider a linear system of the form

$$
\left(\begin{array}{cc}
A & B^{T} \\
B & 0
\end{array}\right)\left(\begin{array}{l}
x \\
y
\end{array}\right)=\left(\begin{array}{l}
f \\
g
\end{array}\right)
$$

with stiffnes matrices $A$ and $B$ as in (4.3). We consider a method of an inexact Uzawa type as analyzed in [3], [23]. For this we assume symmetric positive definite preconditioners $Q_{A}$ of $A$ and $Q_{S}$ of the Schur complement $S:=B A^{-1} B^{T}$. We assume that $Q_{A}$ and $Q_{S}$ are scaled such that $Q_{A}-A$ and $Q_{S}-S$ are positive semidefinite. Furthermore, let constants $\sigma_{A}, \sigma_{S} \in[0,1)$ be such that

$$
\begin{aligned}
\left(1-\sigma_{A}\right)\left\langle Q_{A} x, x\right\rangle & \leq\langle A x, x\rangle \quad \text { for all } x \in \mathbb{R}^{n}, \\
\left(1-\sigma_{S}\right)\left\langle Q_{S} y, y\right\rangle & \leq\langle S y, y\rangle \text { for all } y \in \mathbb{R}^{m} .
\end{aligned}
$$

Note that since $Q_{A}$ and $Q_{S}$ are positive definite, such $\sigma_{A}$ and $\sigma_{S}$ always exist. The inexact Uzawa method is as follows: for $x_{0} \in \mathbb{R}^{n}, y_{0} \in \mathbb{R}^{m}$ given, $\left(x_{i}, y_{i}\right), i=$ $1,2, \ldots$ is determined by

$$
\begin{aligned}
& x_{i+1}=x_{i}+Q_{A}^{-1}\left(f-\left(A x_{i}+B^{T} y_{i}\right)\right), \\
& y_{i+1}=y_{i}+Q_{S}^{-1}\left(B x_{i+1}-g\right) .
\end{aligned}
$$

In [3] it is shown that for the error $e_{i}:=\left(\begin{array}{l}x-x_{i} \\ y-y_{i}\end{array}\right)$ the inequality

$$
\left[\left|e_{i}\right|\right] \leq \rho^{i}\left[\left|e_{0}\right|\right] \quad \text { for } i=0,1,2, \ldots
$$

holds, where $[|\cdot|]$ is a suitable problem-dependent norm and

$$
\rho=\frac{\sigma_{S}\left(1-\sigma_{A}\right)+\sqrt{\sigma_{S}^{2}\left(1-\sigma_{A}\right)^{2}+4 \sigma_{A}}}{2} \leq 1-\frac{1}{2}\left(1-\sigma_{S}\right)\left(1-\sigma_{A}\right) .
$$

From these results we see that one obtains fast convergence of the inexact Uzawa method if one uses good preconditioners $Q_{A}$ and $Q_{S}$.

For our analysis we introduce the discrete analog of the quantity $\Gamma$ :

$$
\sqrt{\Gamma_{h}}:=\sup _{v_{h} \in X_{h}, q_{h} \in M_{h}} \frac{b\left(v_{h}, q_{h}\right)}{\left\|v_{h}\right\|_{X}\left\|q_{h}\right\|} .
$$

Lemma 5.1. Let $\hat{M}_{h}$ be the mass matrix as in (4.3) and $Q_{S}:=\Gamma_{h} \hat{M}_{h}$, then $Q_{S}-S$ is positive semidefinite and for $\sigma_{S}:=1-\frac{\gamma_{h}}{\Gamma_{h}}$ the inequality (5.3) holds.

Proof. Note that with $\tilde{B}:=\hat{M}^{-\frac{1}{2}} B$,

$$
\begin{aligned}
\sqrt{\Gamma_{h}} & =\sup _{x \in \mathbb{R}^{n}, y \in \mathbb{R}^{m}} \frac{\langle B x, y\rangle}{\langle A x, x\rangle^{\frac{1}{2}}\langle\hat{M} y, y\rangle^{\frac{1}{2}}} \\
& =\sup _{x \in \mathbb{R}^{n}, y \in \mathbb{R}^{m}} \frac{\langle\tilde{B} x, y\rangle}{\|x\|_{A}\|y\|} .
\end{aligned}
$$

The relations (4.4), (5.6), and Lemma [2.1] imply

$$
\gamma_{h} I \leq \tilde{B} A^{-1} \tilde{B}^{T} \leq \Gamma_{h} I
$$


and thus for $Q_{S}=\Gamma_{h} \hat{M}_{h}$,

$$
\frac{\gamma_{h}}{\Gamma_{h}} Q_{S} \leq S \leq Q_{S}
$$

Thus, $Q_{S}-S$ is positive semidefinite and (5.3) holds with $\sigma_{S}=1-\frac{\gamma_{h}}{\Gamma_{h}}$.

The results in Lemma 5.1 and (5.5) show that for fast convergence of the Uzawa method, it is favourable to have small $\sigma_{S}$ values, i.e., small values for $\Gamma_{h} \gamma_{h}^{-1}$. We now consider the Stokes problem; i.e., in the remainder we assume that the bilinear forms $a$ and $b$ are as in (3.9). We analyze, for $\alpha \in\{0,1\}$, the dependence of $\Gamma_{h} \gamma_{h}^{-1}$ on the parameters $\xi, \nu, h$. We assume that the finite element spaces $X_{h}$ are such that the inverse inequality

$$
\left\|\nabla v_{h}\right\| \leq c_{I} h^{-1}\left\|v_{h}\right\| \quad \text { for all } v_{h} \in X_{h}
$$

holds, with a constant $c_{I}$ that does not depend on $h$.

Lemma 5.2. The following holds:

$$
\begin{aligned}
& \frac{\Gamma_{h}}{\gamma_{h}} \leq \hat{\beta}^{-2} \quad \text { if } \alpha=0, \\
& \frac{\Gamma_{h}}{\gamma_{h}} \leq \hat{\beta}^{-2} \frac{\nu+c_{F}^{2}+\xi}{\nu+c_{I}^{-2} h^{2}+\xi} \quad \text { if } \alpha=1 .
\end{aligned}
$$

Proof. Using the inverse inequality, it follows that

$$
\left\|u_{h}\right\|_{X}^{2}=\nu\left\|\nabla u_{h}\right\|^{2}+\alpha\left\|u_{h}\right\|^{2}+\xi\left\|\operatorname{div} u_{h}\right\|^{2} \geq\left(\nu+\alpha c_{I}^{-2} h^{2}+\xi\right)\left\|\operatorname{div} u_{h}\right\|^{2} .
$$

Hence we get, due to $b\left(v_{h}, q_{h}\right) \leq\left\|\operatorname{div} v_{h}\right\|\left\|q_{h}\right\|$,

$$
\Gamma_{h} \leq \frac{1}{\nu+\alpha c_{I}^{-2} h^{2}+\xi} .
$$

A lower bound for $\gamma_{h}$ is given in 4.10. Now combine these bounds for $\Gamma_{h}$ and $\gamma_{h}$ and take $\alpha \in\{0,1\}$.

From the results in (5.8) and Lemma 5.1 it follows that for $\alpha=0$ and for all $\xi$ values, the scaled mass matrix $Q_{S}=\Gamma_{h} \hat{M}_{h}$ is a good preconditioner for the Schur complement. For this choice we have $1-\sigma_{S}>c_{0}>0$ with a constant $c_{0}$ independent of $\nu$ and $h$.

For the case $\alpha=1$, the result in (5.9) yields

$$
\frac{\Gamma_{h}}{\gamma_{h}}=\mathcal{O}\left(h^{-2}\right) \quad \text { for } h \downarrow 0, \nu \leq h^{2}, \xi=0,
$$

hence a rapid growth for $h \rightarrow 0$ and sufficiently small $\nu$. In this case a simple scaled mass matrix is not appropriate and one needs special preconditioners for the Schur complement as discussed in 2, [11, [5]. For $\alpha=\xi=1$, we have

$$
\frac{\Gamma_{h}}{\gamma_{h}} \leq \frac{2+c_{F}^{2}}{2+c_{I}^{-2} h^{2}} \quad \text { for all } \nu \in(0,1],
$$

and thus for the scaled mass matrix we have (as for the case $\alpha=0) 1-\sigma_{S}>c_{0}>0$ with a constant $c_{0}$ independent of $\nu$ and $h$.

Remark 6 . We apply the robustness result for the case $\alpha=\xi=1$ to the transientlike problem (1.3) using the scaling argument discussed in the introduction. Let

$$
L=\left(\begin{array}{cc}
A & B^{T} \\
B & 0
\end{array}\right)
$$


be the finite element stiffness matrix as in (5.1) corresponding to the original problem (1.1) for the case $\alpha=\xi=1$. Its Schur complement is denoted by $S$. From the analysis above we have that, uniformly for $h, \nu \in(0,1]$, the scaled mass matrix $Q_{S}$ is a good preconditioner for $S$ (Lemma 5.1).

The same finite element discretization applied to problem (1.3) with $\tilde{\xi}=\frac{1}{\Delta t}$, $\tilde{\nu}=\frac{\nu}{\Delta t}$ yields the stiffness matrix

$$
L_{t}=\left(\begin{array}{cc}
\frac{1}{\Delta t} A & B^{T} \\
B & 0
\end{array}\right)
$$

with Schur complement $S_{t}=\Delta t S$. Hence, uniformly for $h, \nu \in(0,1]$ and $\Delta t \in$ $(0,1]$, the scaled mass matrix $\Delta t Q_{S}$ is a good preconditioner for $S_{t}$.

Besides a good preconditoner $Q_{S}$ for the Schur complement, one also needs a preconditioner $Q_{A}$ of $A$. The stiffness matrix $A$ is given by

$\langle A x, y\rangle=\nu\left(\nabla J_{X} x, \nabla J_{X} y\right)+\alpha\left(J_{X} x, J_{X} y\right)+\xi\left(\operatorname{div} J_{X} x, \operatorname{div} J_{X} y\right) \quad$ for all $x, y \in \mathbb{R}^{n}$, with $J_{X}: \mathbb{R}^{n} \rightarrow X_{h}$ the finite element isomorphism. For the case $\xi=0$ one can use a multigrid method as a preconditioner for $A$. It is known ([13]) that a standard multigrid method results in a precondioner $Q_{A}$ of $A$ with $\left(1-\sigma_{A}\right) Q_{A} \leq A \leq Q_{A}$ with a constant $\sigma_{A}<1$ independent of $h, \nu \in(0,1], \alpha \geq 0$. Hence, for the case $\xi=0$ (no stabilization) a good (i.e., robust w.r.t. variation in the parameters) preconditioner for $A$ is known.

Opposite to this, a robust preconditioner $Q_{A}$ of $A$ for the case with $\nabla$ divstabilization $(\xi=1)$ is an open problem. For $\xi=1$ additional stiffness is introduced due to the div operator which in general has a large kernel. A suitable robust preconditioner for this case is a topic of current research. Techniques presented in [17] may be applicable in this setting.

Summarizing, we have the following results concerning the convergence of the inexact Uzawa method. For fast convergence one needs good (robust w.r.t. variation in parameters) preconditioners of $S$ and $A$. We restrict ourselves to the cases $\xi \in\{0,1\}, \alpha \in\{0,1\}$ :

- $\xi=\alpha=0$ : the scaled mass matrix $Q_{S}$ is a good preconditioner for $S$. Multigrid is a good preconditioner for $A$.

- $\xi=0, \alpha=1$ : the preconditoner $Q_{S}$ is not robust (for $\nu \leq h^{2} \downarrow 0$ ). More sophisticated preconditioning techniques which lead to robust preconditoners, like the methods in [2, 11], should be used. Multigrid is a good preconditioner for $A$.

- $\xi=1, \alpha \in\{0,1\}$ : the scaled mass matrix $Q_{S}$ is a good preconditioner for $S$. A robust preconditioner for $A$ is not yet known.

\section{Numerical EXPERIMENTS}

As a test example we take the Stokes equations (3.3) on $\Omega=(0,1) \times(0,1)$. The right-hand side $f$ is taken such that the continuous solution is

$$
\begin{aligned}
u_{1}(x, y) & =4(2 y-1) x(1-x), \\
u_{2}(x, y) & =-4(2 x-1) y(1-y), \\
p(x, y) & =3\left(x^{3}+y^{3}-0.5\right) .
\end{aligned}
$$

Note that the continuous solution is independent of the parameters $\nu, \alpha$. For the discretization we use a uniform triangulation with mesh size $h$. For $X_{h} \times M_{h}$ we 
take the P1isoP2-P0 finite element pair (piecewise-constant pressure and piecewiselinear continuous velocity on a once refined triangulation). This pair is known to be LBB stable, i.e., condition (4.1) holds.

The discrete problem is solved using the inexact Uzawa method from the previous section. For the Schur complement preconditioner $Q_{S}$ we take the pressure mass matrix (indentity in our case) scaled by $\left(\nu+c_{I}^{-2} h_{u}^{2} \alpha+\xi\right)^{-1}$, cf. (5.10). Here $h_{u}=\frac{h}{2}$ is the size of velocity element. We take $c_{I}=2 \sqrt{2}$ for the constant from (5.7). This value can be found from a spectral upper bound for the discrete Laplacian in a unit square. If a reasonable bound for $c_{I}$ is not available, the term $c_{I}^{-2} h_{u}^{2} \alpha$ can be ignored. This will result in a smaller value of $1-\sigma_{S}$ in (5.3).

For the preconditioner $Q_{A}$ we use a standard multigrid V-cycle method. The prolongations and restrictions are the canonical ones. We used two pre- and two post-smoothings with a symmetric block-Gauss-Seidel iteration.

For the stopping criterion in the inexact Uzawa iteration (5.4), we take a reduction of the relative residual by at least a factor $10^{5}$. To illustrate the performance of the solver, we show in Tables 6.16.2 $N_{\text {iter }}$, the total number of inexact Uzawa iterations required to satisfy the stopping criterion. As was discussed in the previous section, the quantities $\sigma_{A}$ and $\sigma_{S}$ defined in 5.2) - (5.3) characterize the rate of convergence of (5.4). An estimate for $\sigma_{S}$ was obtained in Lemma 5.1. The value of $\sigma_{A}$ depends on the performance of the multigrid method for the velocity problem, which defines $Q_{A}$. The values $\psi_{c d}$ in the tables are estimates for the contraction number of the multigrid method. Since we use two iterations of the multigrid method for solving the velocity problem, we have $\sigma_{A} \approx \psi_{c d}^{2}$.

Tables 6.1 and 6.2 present error norms and convergence data for the problem with $\alpha=0$ solved on meshes with $h=\frac{1}{32}$ and $h=\frac{1}{64}$, respectively. For the problem without $\nabla$ div stabilization $(\xi=0)$, the $O\left(\nu^{-1}\right)$ dependence of the error in velocity as predicted by our theory is clearly seen. For the stabilized problem $(\xi=0.1)$, this dependence is much milder (analytical estimate was $\left.O\left(\nu^{-\frac{1}{2}}\right)\right)$. Note

TABle 6.1. Dependence on $\nu: h=1 / 32, \alpha=0$

\begin{tabular}{ccccc}
\hline \multirow{4}{*}{ Parameter } & & \multicolumn{3}{c}{ viscosity } \\
\cline { 3 - 5 } & Quantity & 1 & $10^{-2}$ & $10^{-4}$ \\
\hline \multirow{4}{*}{$\xi=0$} & $\left\|\nabla\left(u-u_{h}\right)\right\|$ & $5.0 e-2$ & $4.4 e-0$ & $4.0 e+2$ \\
& $\left\|u-u_{h}\right\|$ & $4.1 e-4$ & $3.7 e-2$ & $3.7 e-0$ \\
& $\left\|p-p_{h}\right\|$ & $3.5 e-2$ & $3.5 e-3$ & $3.5 e-3$ \\
& $N_{\text {iter }}$ & 38 & 38 & 38 \\
& $\psi_{c d}$ & 0.06 & 0.06 & 0.06 \\
\hline \multirow{4}{*}{$\xi=0.1$} & $\left\|\nabla\left(u-u_{h}\right)\right\|$ & $4.7 e-2$ & $3.8 e-1$ & $5.5 e-1$ \\
& $\left\|u-u_{h}\right\|$ & $3.8 e-4$ & $3.4 e-3$ & $5.0 e-3$ \\
& $\left\|p-p_{h}\right\|$ & $3.8 e-2$ & $3.8 e-3$ & $3.4 e-3$ \\
& $N_{\text {iter }}$ & 36 & 13 & 312 \\
& $\psi_{c d}$ & 0.06 & 0.30 & 0.96 \\
\hline
\end{tabular}

$N_{\text {iter }}$ - total number of inexact Uzawa iterations

$\psi_{c d}$ - convergence factor in the MG-preconditioner for $A$ 
TABle 6.2. Dependence on $\nu: h=1 / 64, \alpha=0$

\begin{tabular}{ccccc}
\hline \multirow{2}{*}{ Parameter } & Quantity & 1 & $10^{-2}$ & $10^{-4}$ \\
\cline { 3 - 5 } & $\left\|\nabla\left(u-u_{h}\right)\right\|$ & $2.5 e-2$ & $2.0 e-0$ & $2.0 e+2$ \\
& $\left\|u-u_{h}\right\|$ & $1.0 e-4$ & $9.5 e-3$ & $9.5 e-1$ \\
$\xi=0$ & $\left\|p-p_{h}\right\|$ & $1.7 e-2$ & $1.2 e-3$ & $1.2 e-3$ \\
& $N_{\text {iter }}$ & 39 & 36 & 34 \\
& $\psi_{c d}$ & 0.06 & 0.06 & 0.06 \\
\hline \multirow{4}{*}{$\xi=0.1$} & $\left\|\nabla\left(u-u_{h}\right)\right\|$ & $2.4 e-2$ & $1.8 e-1$ & $2.5 e-1$ \\
& $\left\|u-u_{h}\right\|$ & $9.8 e-5$ & $8.5 e-4$ & $5.0 e-3$ \\
& $\left\|p-p_{h}\right\|$ & $1.9 e-2$ & $1.9 e-3$ & $1.7 e-3$ \\
& $N_{\text {iter }}$ & 37 & 12 & 414 \\
& $\psi_{c d}$ & 0.06 & 0.34 & 0.98 \\
\hline
\end{tabular}

$N_{\text {iter }}$ - total number of inexact Uzawa iterations

$\psi_{c d}$ - convergence factor in the MG-preconditioner for $A$

that the error in pressure is insensitive both to viscosity and stabilization, which is in agreement with 4.16) (for $\xi=0$ ) and with (4.13) (for $\xi>0$ ). Comparing results from Tables 6.1 and 6.2 we observe approximately $O(h)$ convergence in velocity gradients and in pressure and $O\left(h^{2}\right)$ in velocity, as expected from theory. The slow convergence of the Uzawa method for stabilized equations with small $\nu$ is caused by the poor convergence of the multigrid method for the velocity problem (see the values of $\psi_{c d}$ ). It is clear that in practice (for small $\nu$ values) this multigrid solver

TABle 6.3. Dependence on $\nu: h=1 / 64, \alpha=1$

\begin{tabular}{ccccc}
\hline \multirow{4}{*}{ Parameter } & Quantity & 1 & $10^{-2}$ & $10^{-4}$ \\
\cline { 3 - 5 } & $\left\|\nabla\left(u-u_{h}\right)\right\|$ & $2.5 e-2$ & $2.0 e-0$ & $1.7 e+2$ \\
& $\left\|u-u_{h}\right\|$ & $1.0 e-4$ & $9.3 e-3$ & $7.6 e-1$ \\
$\xi=0$ & $\left\|p-p_{h}\right\|$ & $1.7 e-2$ & $2.8 e-3$ & $1.6 e-1$ \\
& $N_{\text {iter }}$ & 39 & 124 & 3829 \\
& $\psi_{c d}$ & 0.05 & $2.6 e-2$ & $1.7 e-3$ \\
\hline \multirow{4}{*}{$\xi=0.1$} & $\left\|\nabla\left(u-u_{h}\right)\right\|$ & $2.4 e-2$ & $1.9 e-1$ & $3.6 e-1$ \\
& $\left\|u-u_{h}\right\|$ & $9.8 e-5$ & $8.4 e-4$ & $1.6 e-3$ \\
& $\left\|p-p_{h}\right\|$ & $1.9 e-2$ & $1.9 e-3$ & $1.7 e-3$ \\
& $N_{\text {iter }}$ & 37 & 20 & 217 \\
& $\psi_{c d}$ & 0.05 & 0.34 & 0.97 \\
\hline & & & &
\end{tabular}

$N_{\text {iter }}$ - total number of inexact Uzawa iterations

$\psi_{c d}$ - convergence factor in the MG-preconditioner for $A$ 
should not be used. An efficient alternative for this multigrid solver is a topic of current research.

In the Table 6.3 we show results for $\alpha=1$. In this case, as predicted by the results in (4.14), (4.15), for small $\nu$ values due to the $\nabla$ div term, one obtains much smaller discretization errors both for pressure and velocity. We also observe a strong deterioration of the convergence of the inexact Uzawa method for the case $\xi=0$. This is probably due to the fact that for $\nu \rightarrow 0, h \rightarrow 0$ the scaled mass matrix is not a good preconditioner for the Schur complement (cf. (5.11) and the discussion in Section [5).

\section{ConCLUding REMARKS}

We summarize the effect of $\nabla$ div stabilization. For the continuous problem, the stabilization does not change the solution but enhances the stability of the corresponding bilinear form. For the case $\xi=\xi_{0}>0$, the problem is uniformly (for $\nu \downarrow 0)$ well-posed in the natural norm (3.14).

For finite element discretization, stabilization changes the discrete solution and results in better error bounds. For example, for the P1isoP2/P0 finite elements we have (with $\alpha=0$ ) the sharp bound $\left\|\nabla\left(u-u_{h}\right)\right\| \leq C h\left(\|u\|_{2}+\nu^{-1}\|p\|_{1}\right.$ ) for $\xi=0$ and $\left\|\nabla\left(u-u_{h}\right)\right\| \leq C \nu^{-\frac{1}{2}} h\left(\|u\|_{2}+\|p\|_{1}\right)$ for $\xi=1$. Our analysis does not yield sharp discretization error bounds for a time-discretized problem as in (1.3) if $\Delta t \downarrow 0$.

The $\nabla$ div stabilization influences the convergence behaviour of the inexact Uzawa iterative solver. The main results are summarized at the end of Section 5

\section{ACKNOWLEDGMEnts}

Part of this work was done by the first author while he was visiting Vanderbilt University in the fall of 2001. For this author the work was also supported partially by the Russian Foundation for Basic Research grant No. 02-01-0615 linked to project 02-01-00592.

The authors thank the referees for several comments which lead to a considerably improved presentation.

\section{REFERENCES}

1. Bank, R.E., Welfert, B.D., and Yserentant, H. A class of iterative methods for solving saddle point problems, Numer. Math., 56 (1990), pp. 645-666. MR 91b:65035

2. Bramble, J.H., And Pasciak, J.E. Iterative techniques for time dependent Stokes problems, Comput. Math. Appl. 33 (1997), pp. 13-30. MR 98e:65091

3. Bramble, J.H., Pasciak, J.E., And Vassilev, A.T. Analysis of the inexact Uzawa algorithms for saddle point problems, SIAM J. Numer. Anal., 33 (1997) , pp. 1072-1092. MR 98c:65182

4. Bychenkov, Yu.V. And Chizonkov, E.V. Optimization of one three-parameter method of solving an algebraic system of Stokes type, Russ. J. Numer. Anal. Math. Model, 14 (1999), pp. 429-440. MR 2000h:65062

5. Cahouet, J., and Chabard, J.P. Some fast 3D finite element solvers for the generalized Stokes problem, Int. J. Numer. Methods Fluids., 8 (1988), pp. 869-895. MR 89i:76005

6. Chizhonkov, E.V., And Olshanskit, M.A., On the domain geometry dependence of the LBB condition, Math. Model. Anal. Numer. (M2AN), 34 (2000) pp. 935-951. MR 2002c:65203

7. Fujita, H, And Suzuki, T. Evolution problems, in: Handbook of numerical analysis (eds. P.G. Ciarlet and J.L. Lions), Vol. 2, pp. 789-928, North-Holland, Amsterdam, 1991.

8. Girault, V., And Raviart, P.-A. Finite element methods for Navier-Stokes equations, Springer, Berlin, 1986. MR 88b:65129 
9. Glowinski, R., And Le Tallec, P. Augmented lagrangian and operator splitting methods in nonlinear mechanics, SIAM, Studies in Applied Mathematics, Philadelphia, 1989. MR 91f: 73038

10. Kobelkov G.M. On solving the Navier-Stokes equations at large Reynolds numbers, Russ. J. Numer. Anal. Math. Model, 10 (1995), pp. 33-40. MR 95m:65167]

11. Kobelkov, G.M. and Olshanskit, M.A. Effective preconditioning of Uzawa type schemes for a generalized Stokes problem, Numer. Math., 86 (2000), pp. 443-470. MR 2001j:65168

12. OlshanskiI, M.A. A low order Galerkin finite element method for the Navier-Stokes equations of steady incompressible flow: a stabilization issue and iterative methods, Comput. Meth. Appl. Mech. Eng., 191 (2002), pp. 5515-5536.

13. Olshanskit, M.A. And Reusken, A. On the convergence of a multigrid algorithm for linear reaction-diffusion problems, Computing, 65 (2000), pp. 193-202. MR 2001m:65186

14. Quarteroni, A. And VAldi, A. Numerical approximation of partial differential equations Springer, Berlin 1997. MR 95i:65005

15. Roos, H.-G., Stynes, M., And Tobiska, L. Numerical methods for singularly perturbed differential equations: convection diffusion and flow problems Springer Series in Computational Mathematics, 24. Springer-Verlag, Berlin, 1996. MR 99a:65134

16. Rusten, T., And Winther, R. A preconditioned iterative method for saddlepoint problems SIAM J. Matrix Anal. Appl. 13 (1992), pp. 887-904. MR 93a:65043

17. SCHÖBERL, J. Multigrid methods for a parameter dependent problem in primal variables, Numer. Math., 84 (1999), pp. 97-119. MR 2001a:65147

18. Silvester, D., And Wathen, A. Fast iterative solution of stabilised Stokes systems. Part II: using general block preconditioners, SIAM J. Numer. Anal., 31 (1994), pp. 1352-1367. MR 95g:65132

19. ThOMEe, V. Galerkin finite element methods for parabolic problems, Springer, Berlin, 1984. MR 86k:65006

20. Tobiska, L., And Verfürth, R. Analysis of a streamline diffusion finite element method for the Stokes and Navier-Stokes equations, SIAM J. Numer. Anal., 33 (1996), pp.107-127. MR 97e:65133

21. Vassilevski, P.S., And Lazarov, R.D. Preconditioning mixed finite element saddle-point elliptic problems, Numer. Linear Alg. Appl., 3 (1996), pp. 1-20. MR 96m:65111

22. VERFüRTH, R. A combined conjugate gradient-multigrid algorithm for the numerical solution of the Stokes problem IMA J. Numer. Anal. 4 (1984), pp. 441-455. MR 86f:65200

23. Zulehner, W. Analysis of iterative methods for saddle point problems: A unified approach, Math. Comp. 71 (2001), pp. 479-505. MR 2003f:65183

Dept. Mechanics and Mathematics, Moscow State University, Moscow 119899, Russia E-mail address: ay@olshan.msk.ru

Institut für Geometrie und Praktische Mathematik, RWTH-Aachen, D-52056 Aachen, Germany

E-mail address: reusken@igpm.rwth-aachen.de 